\title{
Mechanical and Thermal Stresses in an HPDC Machine Plunger During the Dosing and Filling Process
}

\author{
Jacek Jankowski ${ }^{1 *}$, Dariusz Kołakowski ${ }^{2}$, Bogusław Piotr Pisarek ${ }^{2}$ \\ 1 Department of Strength of Materials, Lodz University of Technology, 1/15 Stefanowskiego str., 90-924 Łódź, \\ Poland \\ 2 Department of Materials Engineering and Production Systems, Lodz University of Technology, 1/15 \\ Stefanowskiego str., 90-924 Łódź, Poland \\ * Corresponding author's e-mail: jacek.jankowski@p.lodz.pl
}

\begin{abstract}
Distributions of mechanical and thermal stresses in a plunger of the high pressure die casting machine with a horizontal shot sleeve have been considered for a flat thermal stationary problem. An axial pressure of the plunger pressing in the technological process has been accounted for as well. Principal and equivalent stresses have been determined within two different hypotheses on the strength of materials, including one theory covering various allowable stresses in tension and compression. Four plunger material variants have been analysed.
\end{abstract}

Keywords: plunger, HPDC machine, mechanical and thermal stresses, flat thermal stationary state, equivalent stresses, different tensile and compression strength.

\section{INTRODUCTION}

High pressure die casting (HPDC) of aluminium and copper alloys is the primary technology in manufacturing products for home appliances, automotive industry, etc. A detailed analysis of the technological process during high pressure die casting is very complex indeed due to mechanical and thermal fatigue, creeping, erosion, cavitation, dissolution of mould and metal components, adhesion. The state of mechanical and thermal stresses in a HPDC machine plunger, covering the product pressing stage, has been determined. One of reasons standing behind too early destruction of plungers is an interaction of thermal internal stresses with longitudinal stresses generated by the cyclic process during their operation. In the fixed production cycle of die casting, after the start-up phase, it can be assumed that thermal stresses do not alter during one operation cycle due to a high heat capacity of the mould.
In [4] devoted to analysis of the mechanical and thermal stress state in a plunger of the cold-chamber HPDC machine with a horizontal shot sleeve, temperature distributions in the shot sleeve were discussed on the basis of [6, 7]. In [4], the stress state was considered on the assumption of a homogenous temperature field in the plunger surroundings. Stress distributions were analysed for maximal temperature values on the contact surface of the machine plunger and the shot sleeve, that is to say, on the plunger outer surface. The temperature on the plunger inner surface is minimal, as the plunger interior is cooled with a cooling agent forced circulation. For comparison, calculations were conducted for four different materials used for plunger in cold-chamber HPDC machines, namely: grey pearlitic cast iron, high silicone bronze, beryllium bronze and aluminium-iron-nickel bronze which is the source alloy for newly developed complex bronzes of high wear resistance and enhanced strength. 
In the present work, a distribution of stress state components for the non-homogenous stationary temperature field, i.e., for the conditions close to actual operation conditions of the plunger, is dealt with. The whole cycle including the time for dosing of molten metal lasts less than 3 minutes, whereas the plunger loading is shorter than $30 \mathrm{~ms}$ (filling). In the case of the HPDC machine plunger, pressing pressure is a compressive axial load equal even up to $100 \mathrm{MPa}$, whereas the maximum load is equal to zero during dosing stage. From the viewpoint of fatigue resistance, it is a negative pulsating cycle.

One of most important tribological issues is to determine maximal mechanical and thermal stresses, including equivalent stresses, according to the Huber-Mises-Hencky hypothesis. The materials the plungers are made of can be characterized by various tensile and compression strengths. The compression strengths $R_{c}$ are significantly higher than the tensile strengths $R_{m}$. Thus, the allowable stresses in compression $\mathrm{k}_{\mathrm{c}}$ are higher than the allowable stresses in tension $k_{r}$ (i.e., $k_{c}>k_{r}$ ). In the considerations presented here, a tensile strength to compressive strength ratio: $z=R_{c} / R_{m}>1$ (or $k_{c} / k_{r}>1$ ) has been introduced. On the basis of the results presented in [4], a strength hypothesis that accounts for various maximal stresses in compression and tension, that is to say, the so-called Burzynski hypothesis, has been assumed.

In the technological process in the plane transverse to the plunger axis, only radial, circumferential and tangential thermal stresses, caused by a temperature difference inside and outside the plunger, occur, whereas mechanical stresses are present along the longitudinal direction. Thermal stresses alter circumferentially from the maximal ones in the bottom part of the machine slot sleeve to the minimal ones in the upper part of the sleeve. Therefore, such a temperature distribution for the flat stationary state and longitudinal stresses caused by the plunger pressing pressure in the technological process have been considered in the present study.

An analysis of the plunger/sleeve operation discussed here is based on a representation of the actual die casting process that takes place with a Buehler machine in the WIFAMA-PREXER company located in Lodz [4].

\section{FORMULATION OF THE PROBLEM}

In the present study, a temperature distribution in the HPDC machine plunger that is closer the actual one has been analysed. For the thickwalled plunger/sleeve, a cylindrical system of coordinates has been assumed. For the flat thermal stationary problem, the following temperature distribution is taken (Fig. 1):

$$
T(r, \varphi)=\bar{T}(r)+\widetilde{T}(r, \varphi)=\bar{T}(r)+\hat{T}(r) \cos \varphi
$$

where: $0 \leq \varphi \leq 2 \pi, a \leq r \leq b$

with the boundary conditions:

$$
\begin{aligned}
\bar{T}(r=a)=\bar{T}_{a}, \bar{T}(r=b) & =\bar{T}_{b}, \hat{T}(r=a)=0, \\
\hat{T}(r=b) & =\hat{T}_{b}
\end{aligned}
$$

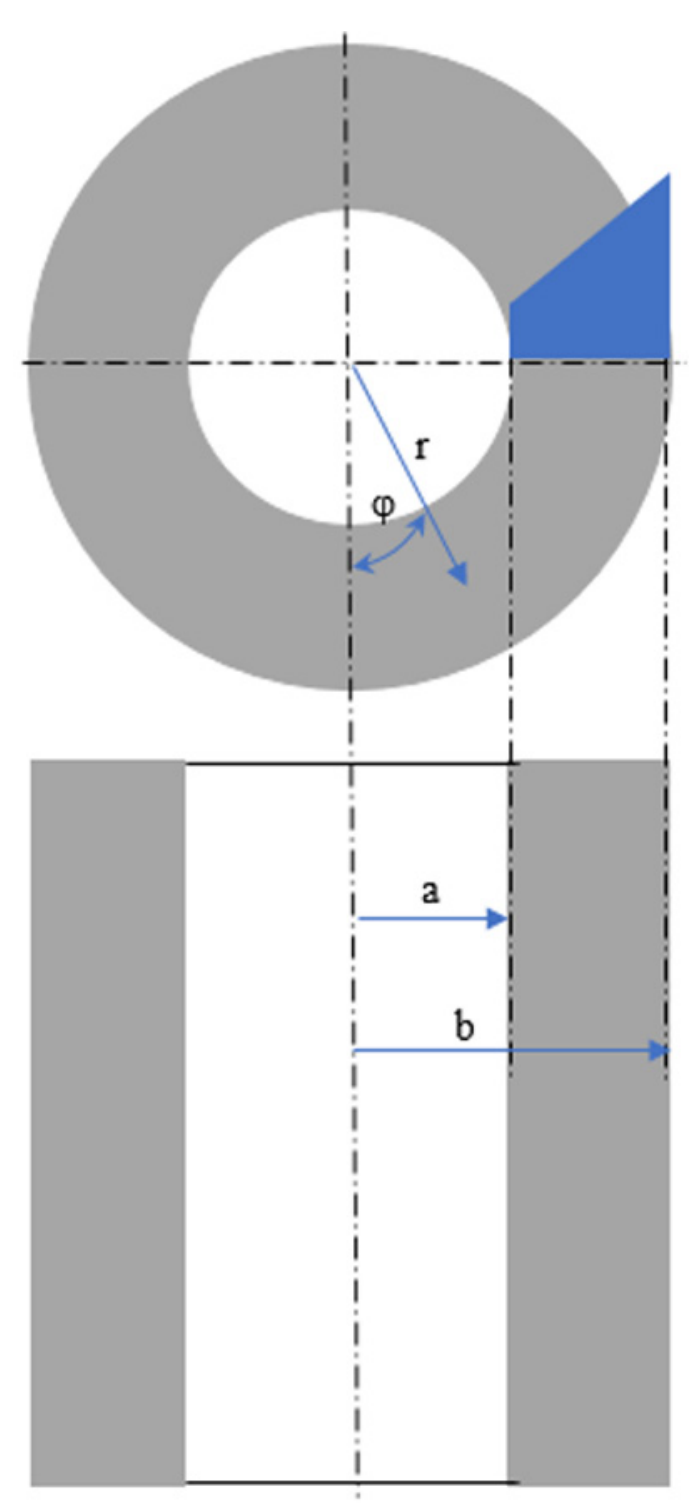

Fig. 1. Long thick-walled cylindrical tube - plunger 
thus, according to (1) and (2), we have:

$$
T(r=a, \varphi)=\bar{T}_{a} \text { and } T(r=b, \varphi)=\bar{T}_{b}+\hat{T}_{b} \cos \varphi
$$

In order to derive relationships describing temperature distributions as well as mechanical and thermal stresses for the flat stationary problem, the following has been assumed $[2,5,8]$ :

1. Kelvin's phenomenon is neglected (an increase in temperature is accompanied by material strains);

2. There is no heat source (i.e., $Q=0$ );

3. A temperature field is determined independently of stresses and strains from the classical equation of conductivity, i.e., for the stationary problem;

4. A temperature curve has been assumed for the flat problem and it does not depend on the coordinate $z$ along the sleeve axial direction;

5. Thermal stresses are caused by the time-constant temperature field;

6 . Elastic and thermal properties of the pipe material do not depend on temperature;

7. The longitudinal stresses $\sigma_{z}$ do not depend on temperature and their stable uniform distribution has been assumed $[1,4]$.

The basic equations for the flat problem $[2,8]$ are expressed with the relationships:

- Equations of heat conductivity in the flat stationary problem:

$$
\frac{\partial^{2} T}{\partial r^{2}}+\frac{1}{r} \frac{\partial T}{\partial r}+\frac{1}{r^{2}} \frac{\partial^{2} T}{\partial \varphi^{2}}=0
$$

- Equation of equilibrium for the flat stress state:

$$
\begin{aligned}
& \frac{\partial \sigma_{r}}{\partial r}+\frac{1}{r} \frac{\partial \sigma_{r \varphi}}{\partial \varphi}+\frac{\sigma_{r}-\sigma_{\varphi}}{r}=0 \\
& \frac{\partial \sigma_{r \varphi}}{\partial r}+\frac{1}{r} \frac{\partial \sigma_{\varphi}}{\partial \varphi}+\frac{2}{r} \sigma_{r \varphi}=0
\end{aligned}
$$

- Geometrical relationships:

$$
\begin{gathered}
\varepsilon_{r}=\frac{\partial u}{\partial r} \quad \varepsilon_{\varphi}=\frac{1}{r} \frac{\partial v}{\partial \varphi}+\frac{u}{r} \\
\gamma_{r \varphi}=\frac{\partial v}{\partial r}-\frac{v}{r}+\frac{1}{r} \frac{\partial u}{\partial \varphi}
\end{gathered}
$$

where: $r$-sleeve radius,

$u$ - radial displacement,

$v$ - circumferential displacement,

$\varepsilon_{r}$ - radial strains,

$\varepsilon_{t}-$ circumferential strains,

$\gamma_{\mathrm{r} \varphi}$ - modal strains, $\sigma_{r}-$ radial stresses,

$\sigma_{\varphi}-$ circumferential stresses,

$\sigma_{r \varphi}-$ tangential stresses,

$a$ - pipe inner radius,

$b$ - pipe outer radius (thus, we have

$a \leq r \leq b)$.

For the flat stress state (i.e., $\sigma_{z}=0$ ), the relationships between stresses and strains take the form:

$$
\begin{aligned}
& \left.\sigma_{r}=\frac{E}{\left(1-v^{2}\right)}\left[\varepsilon_{r}+v \varepsilon_{t}-(1+v) \alpha \Delta T\right]\right) \\
& \sigma_{t}=\frac{E}{\left(1-v^{2}\right)}\left[\varepsilon_{t}+v \varepsilon_{r}-(1+v) \alpha \Delta T\right] \\
& \sigma_{r \varphi}=G \gamma_{r \varphi}=\frac{E}{2(1+v)} \gamma_{r \varphi}
\end{aligned}
$$

where: $E$ - Young's modulus,

$v$ - Poisson's ratio,

$\alpha$ - linear expansion coefficient,

and, moreover

$$
\Delta T=T-T_{a} \text { (where: } T_{a} \leq T \leq T_{b} \text { ) }
$$

For the problem under consideration, the following boundary conditions are assumed:

$$
\begin{aligned}
& \sigma_{r}(r=a)=\sigma_{r}(r=b)=0 \\
& \sigma_{r \varphi}(r=a)=\sigma_{r \varphi}(r=b)=0
\end{aligned}
$$

i.e., the radial stresses $\sigma_{r}$ and the tangential stresses $\sigma_{r \varphi}$ on both plunger/sleeve surfaces are equal to zero.

After substituting (6) into (7), relationships between components of the stress state and components of displacements are obtained:

$$
\begin{aligned}
& \sigma_{r}=\frac{E}{1-v^{2}}\left[u_{, r}+\frac{v}{r} v_{, \varphi}+\frac{v}{r} u-(1+v) \alpha \Delta T\right] \\
& \sigma_{\varphi}=\frac{E}{1-v^{2}}\left[\frac{1}{r} v_{, \varphi}+\frac{1}{r} u+v u_{, r}-(1+v) \alpha \Delta T\right] \\
& \sigma_{r \varphi}=\frac{E}{2(1+v)}\left[v_{, r}+\frac{1}{r} u_{, \varphi}-\frac{v}{r}\right]
\end{aligned}
$$

where the following notations have been used:

$$
\begin{array}{ll}
u_{, r}=\frac{\partial u}{\partial r}, & u_{, \varphi}=\frac{\partial u}{\partial \varphi} \\
v_{, r}=\frac{\partial v}{\partial r} & v_{, \varphi}=\frac{\partial v}{\partial \varphi}
\end{array}
$$


According to the assumed stationary temperature distribution (1), boundary conditions (2) and (9), the solutions for the stress state components are predicted in the form:

$$
\begin{aligned}
& \sigma_{r}(r, \varphi)=\bar{\sigma}_{r}(r)+\tilde{\sigma}(r, \varphi)=\bar{\sigma}_{r}(r)+\hat{\sigma}_{r}(r) \cos \varphi \\
& \sigma_{\varphi}(r, \varphi)=\bar{\sigma}(r)+\tilde{\sigma}(r, \varphi)=\bar{\sigma}_{\varphi}(r)+\hat{\sigma}_{\varphi}(r) \cos \varphi \\
& \sigma_{r \varphi}(r, \varphi)=\tilde{\sigma}(r, \varphi)=\hat{\sigma}_{r \varphi}(r) \sin \varphi
\end{aligned}
$$

and the corresponding displacement state components as, respectively:

$$
\begin{aligned}
& u(r, \varphi)=\bar{u}(r)+\widetilde{u}(r, \varphi)=\bar{u}(r)+\hat{u}(r) \cos \varphi \\
& v(r, \varphi)=\widetilde{v}(r, \varphi)=\hat{v}(r) \sin \varphi
\end{aligned}
$$

After substitution of (12) in equilibrium equations (5), two systems of equations are obtained, namely:

- for the axially symmetrical stress state (i.e., for the state independent of the angle $\varphi$ in (12), discussed in detail in [4])

$$
\frac{d \bar{\sigma}_{r}}{d r}+\frac{\bar{\sigma}_{r}-\bar{\sigma}_{\varphi}}{r}=0
$$

with the following boundary conditions, according to (9) and (12)

$$
\bar{\sigma}_{r}(r=a)=\bar{\sigma}_{r}(r=b)=0
$$

- for the flat stress state (i.e., for the state dependent on the angle $\varphi$ in (12)), they take the form:

$$
\begin{aligned}
& \hat{\sigma}_{r, r}+\frac{1}{r} \hat{\sigma}_{r \varphi, \varphi}+\frac{\hat{\sigma}_{r}-\hat{\sigma}_{\varphi}}{r}=0 \\
& \hat{\sigma}_{r \varphi, r}+\frac{1}{r} \hat{\sigma}_{\varphi, \varphi}+\frac{2}{r} \hat{\sigma}_{r \varphi}=0
\end{aligned}
$$

with the following boundary conditions, according to (9) and (12):

$$
\begin{aligned}
& \hat{\sigma}_{r}(r=a)=\hat{\sigma}_{r}(r=b)=0 \\
& \hat{\sigma}_{r \varphi}(r=a)=\hat{\sigma}_{r \varphi}(r=b)=0
\end{aligned}
$$

In Appendix I, the solutions obtained in [4] for the axially symmetrical state are presented. In Appendix II, a solution to the flat problem of the temperature distribution in the sleeve for $\widetilde{T}(r, \varphi)=\hat{T}(r) \cos \varphi$ is described, whereas a solution to the equilibrium equations for the flat stress state in the sleeve, corresponding to the temperature distribution according to Appendix II, is shown in Appendix III.

For the flat stress state with the components $\tilde{\sigma}_{r}, \widetilde{\sigma}_{\varphi}, \widetilde{\sigma}_{r \varphi}$ (Appendix III), it can be easily noticed that when $\varphi=0$, then according to (12), the stress components $\max \left(\tilde{\sigma}_{r}\right)=\hat{\sigma}_{r}(r)$, $\max \left(\tilde{\sigma}_{\varphi}\right)=\hat{\sigma}_{\varphi}(r)$ and $\tilde{\sigma}_{r \varphi}=0$. When $\varphi=\pi / 2$, then $\max \left(\tilde{\sigma}_{r \varphi}\right)=\hat{\sigma}_{r \varphi}(r)$ and, moreover, $\widetilde{\sigma}_{r}=\widetilde{\sigma}_{\varphi}=0$.

When the stress state components (12) $\bar{\sigma}_{r}, \bar{\sigma}_{\varphi}, \widetilde{\sigma}_{r}, \widetilde{\sigma}_{\varphi}, \widetilde{\sigma}_{r \varphi}$ are determined for the assumed temperature distribution (1), the principal stresses $\sigma_{1}^{*}, \sigma_{2}^{*}$ for the flat stress state should be defined $[1,3,9]$ in order to find equivalent stresses according to the strength hypotheses accepted.

Taking into account relationships (12), the principal stresses are expressed with the following formulas known from the material strength for the Mohr's circle:

$$
\begin{gathered}
\sigma_{1}^{*}=\frac{\bar{\sigma}_{r}+\bar{\sigma}_{\varphi}+\left(\tilde{\sigma}_{r}+\bar{\sigma}_{\varphi}\right) \cos \varphi}{2}+ \\
+\sqrt{\left(\frac{\bar{\sigma}_{\varphi}-\bar{\sigma}_{r}+\left(\tilde{\sigma}_{\varphi}-\bar{\sigma}_{r}\right) \cos \varphi}{2}\right)^{2}+\left(\tilde{\sigma}_{r \varphi} \sin \varphi\right)^{2}} \\
-\sqrt{\left(\frac{\bar{\sigma}_{\varphi}-\bar{\sigma}_{r}+\left(\tilde{\sigma}_{\varphi}-\bar{\sigma}_{r}\right) \cos \varphi}{2}\right)^{2}+\left(\tilde{\sigma}_{r \varphi} \sin \varphi\right)^{2}}
\end{gathered}
$$

In the case when, analogously as in [4], the maximal pressing pressure of the plunger/sleeve, i.e., the longitudinal stresses $\sigma_{z}$ (or the so-called axial ones), is considered during the process, we have a three-directional stress state (3D). According to what has been assumed in (7), a relationship for the longitudinal principal stress $\sigma_{3}^{*}=\sigma_{z}$ holds.

Knowing the principal stresses $\sigma_{1}^{*}, \sigma_{2}^{*}, \sigma_{3}^{*}$, it is possible to determine equivalent stresses for the two hypotheses under consideration in the present study (see Appendix IV) (cf. [4] as well).

\section{ANALYSIS OF THE RESULTS}

To analyse in detail the stress state in the plunger/sleeve, a special code, whose basic assumptions and formulas are presented in this study, has been written. This code determines components of the stress state, principal stresses and equivalent stresses for mechanical and thermal loads for the two strength theories, namely: the Huber-Mises-Hencky hypothesis and the Burzynski hypothesis.

Detailed computations have been conducted for the following input data referring to actual conditions present during the manufacturing 
process, in a Buehler HPDC machine based in the WIFAMA-PREXER company, namely:

- constant external temperature of the plunger $\overline{T_{b}}=250^{\circ} \mathrm{C}$;

- amplitude of variability in the plunger external temperature $\hat{T}_{b}=50^{\circ} \mathrm{C}$

- plunger inner temperature $\quad \overline{T_{a}}=150^{\circ} \mathrm{C}$;

- plunger outer diameter $\quad D^{a}=2 b=70 \mathrm{~mm}$;

- plunger inner diameter $\mathrm{d}=2 \mathrm{a}=42 \mathrm{~mm}$;

- maximal plunger pressing pressure $\mathrm{p}_{\mathrm{z}}=\sigma_{\mathrm{z}}=-72 \mathrm{MPa}$;

- ratio of various material strengths $z=k_{c} / k_{r}\left(R_{c} / R_{m}\right) \quad \mathrm{z}=1.5$.

Like in [4], it has been assumed that the plunger is made of one of four variants of materials characterized by material constants listed in Table 1. In the computations, the index notations for the given material have been assumed as in Table 1.

In the thermal problem, the stress state components for the axially symmetrical state (A1.5) $\bar{\sigma}_{r}, \bar{\sigma}_{\varphi}$ and for the flat stress state (A3.19) $\widetilde{\sigma}_{r}, \widetilde{\sigma}_{\varphi}, \widetilde{\sigma}_{r \varphi}$ are in a direct proportion to the product $E \alpha$, which has been additionally listed in Table 1. The stress state components depend also on a temperature difference between the outer surface of the plunger/sleeve and its cooled inside. It should be remembered that for the flat stress state, according to (1) and (2), we have $\widetilde{T}(r=a, \varphi)=\hat{T}(r=a) \cos \varphi=0$.

For the 3D mechanical and thermal problem, constant longitudinal stresses in compression (the so-called axial stresses) $\sigma_{3}^{*}=\sigma_{z}$ have been assumed.

In Fig. 2, a plot of the stresses $\bar{\sigma}_{r}$ as a function of the radius $r$ is depicted for the 4 plunger material variants listed in Table 1 and for the axially symmetrical state. Maximal stress values $\bar{\sigma}_{r}$ occur for variant 4 , then for variants 3 and 1 , and the least ones for variant 2 , as can be expected due to the quantity $E \alpha$. Maximal stresses do not exceed $15 \mathrm{MPa}$. According to the boundary conditions, the stresses $\bar{\sigma}_{r}$ for $r=a$ and $r=b$ equal zero. In Fig. 3, alternations in the stresses $\bar{\sigma}_{\varphi}$ versus the radius $r$ for 4 material variants have been presented, also for the axially symmetrical state. Maximal stresses in tension occur on the plunger inner surface $(r=a)$ and are less than $140 \mathrm{MPa}$ for variant 4 , whereas maximal stresses in compression are on the plunger outer surface $(r=b)$ and are less than $100 \mathrm{MPa}$. For $\mathrm{r} \approx 27 \mathrm{~mm}$, the stresses $\bar{\sigma}_{\varphi}$ are equal to zero. A sequence of variants is the same as in Fig. 2.

In Figs. 4 and 5, plots of the stress components $\hat{\sigma}_{r}, \hat{\sigma}_{r \varphi}, \hat{\sigma}_{\varphi}$ as a function of the radius $r$ for an arbitrary value of the angle $\varphi$ are depicted for the flat thermal state. Stresses are determined for a temperature difference between the outer and inner surface equal to $50{ }^{\circ} \mathrm{C}$ and, therefore, are less than stresses for the axially symmetrical state. According to (A3.6), the stress components are equal, i.e., $\hat{\sigma}_{r}=\hat{\sigma}_{r \varphi}$. In Fig. 4 , the maximal stress values $\hat{\sigma}_{r}, \hat{\sigma}_{r \varphi}$ are equal to approx. $5 \mathrm{MPa}$ at most, whereas in Fig. 5 the maximal stresses in tension $\hat{\sigma}_{\varphi}$ are equal to about $50 \mathrm{MPa}$ on the inner surface, while the stresses in compression are equal to approx. $30 \mathrm{MPa}$ outside the plunger. A sequence of alternations in the stress values is identical as in Figs. 2 and 3.

In the next stage, the principal stresses $\sigma_{1}^{*}, \sigma_{2}^{*}, \sigma_{3}^{*}$ and the equivalent stresses $\sigma_{H M H}$ (according to the HMH hypothesis) and $\sigma_{B}$ (according to the Burzynski hypothesis) are determined, respectively, for the $3 \mathrm{D}$ stress state on the assumption that $\sigma_{z}=-72 \mathrm{MPa}$. The stresses $\sigma_{1}^{*}, \sigma_{2}^{*}, \sigma_{3}^{*}$ and $\sigma_{H M H}, \sigma_{B}$ should be determined for the given value of the angle $\varphi$. The maximal values of these components occur for $\varphi=0$, and the lowest ones for $\varphi=180^{\circ}$, whereas they are intermediate for the angle $\varphi=90^{\circ}$.

Further on, in Figs. 6-13, distributions of the equivalent stresses $\sigma_{H M H}$ and $\sigma_{B}$ for, respectively, variants $1-4$ versus the radius $r$, are shown. It should be noticed that when the stresses in compression $\sigma_{z}$ are assumed, the stresses $\sigma_{B}$ decrease with respect to $\sigma_{H M H}$ (for a more detailed analysis, see Appendix IV).

Table 1. Plunger material characteristics

\begin{tabular}{|c|l|c|c|c|c|c|c|c|}
\hline \multirow{2}{*}{ Variants } & \multirow{2}{*}{ Materials } & $E$ & $v$ & $\alpha$ & $E \alpha$ & $R_{m}$ & $R_{c}$ & $z$ \\
\cline { 3 - 9 } & & $\mathrm{GPa}$ & - & $10^{-6} \mathrm{~K}^{-1}$ & $\mathrm{~Pa} / \mathrm{K}$ & $\mathrm{MPa}$ & $\mathrm{MPa}$ & - \\
\hline 1 & CuAl10Fe5Ni5 & 110 & 0.34 & 16.2 & 1782 & 620 & 938 & 1.5 \\
\hline 2 & 50 standard grey iron & 145 & 0.29 & 10.8 & 1566 & 362 & 1130 & 1.5 \\
\hline 3 & High silicon bronze A H-01 & 105 & 0.346 & 18.0 & 1890 & 550 & & 1.5 \\
\hline 4 & Ampcoloy A 89 beryllium copper & 135 & 0.34 & 17.2 & 2322 & 740 & & 1.5 \\
\hline
\end{tabular}




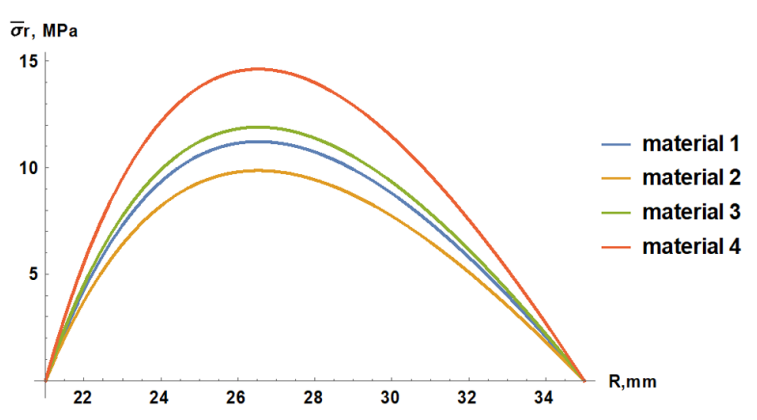

Fig. 2. Plots of the stresses $\bar{\sigma}_{r}$ as a function of the radius for the axially symmetrical thermal problem

One can see in Fig. 6 that the maximal stresses $\sigma_{H M H}$ are $190 \mathrm{MPa}$ for $\mathrm{r}=\mathrm{a}$, and for $\mathrm{r}=\mathrm{b}$ they are about $110 \mathrm{MPa}$ at $\varphi=0$. For the angle $\varphi=180^{\circ}$, they are equal to $120 \mathrm{MPa}$ and $64 \mathrm{MPa}$, respectively. For the angle $\varphi=90^{\circ}$, values of stresses are intermediate. In Fig. 7, a change in the stresses $\sigma_{B}$ is similar, but their values become lower. The maximal stresses $\sigma_{B}$ are about $210 \mathrm{MPa}$ for $\mathrm{r}=\mathrm{a}$, whereas for $\mathrm{r}=\mathrm{b}$ they exceed $70 \mathrm{MPa}$ at $\varphi=0$. On the outer surface, the stresses $\sigma_{B}$ decrease by approx. $30 \mathrm{MPa}$ with respect to $\sigma_{H M H}$, and on the inner surface they are less by approx. $20 \mathrm{MPa}$, correspondingly. A higher reduction in the stresses $\sigma_{B}$ on the outer surface follows from the stresses in compression $\bar{\sigma}_{\varphi}$ and $\hat{\sigma}_{\varphi}$. For the angles $\varphi=0 ; 90^{\circ} ; 180^{\circ}$ and $\mathrm{r} \approx 29 \mathrm{~mm}$, the stresses $\sigma_{H M H}$ differ inconsiderably. Similar relations hold for the stresses $\sigma_{B}$.

In Figs. 8 and 9, similar relationships occur between $\sigma_{H M H}$ and $\sigma_{B}$ for variant 2, in Figs. 10 and 11 for variant 3, and in Figs. 12 and 13 for variant 4 , respectively. As expected, the maximal stresses $\sigma_{H M H}$ and $\sigma_{B}$ occur for variant 4 . For the angle $\varphi=0$ and $\mathrm{r}=\mathrm{a}$, we have $\sigma_{H M H}=231 \mathrm{MPa}$, $\sigma_{B}=212 \mathrm{MPa}$, whereas for $r=b, 111 \mathrm{MPa}$ and $74 \mathrm{MPa}$, correspondingly.

The minimal values $\sigma_{H M H}$ and $\sigma_{B}$ occur for variant 2 . In this case, we have $\sigma_{H M H}=174 \mathrm{MPa}$,

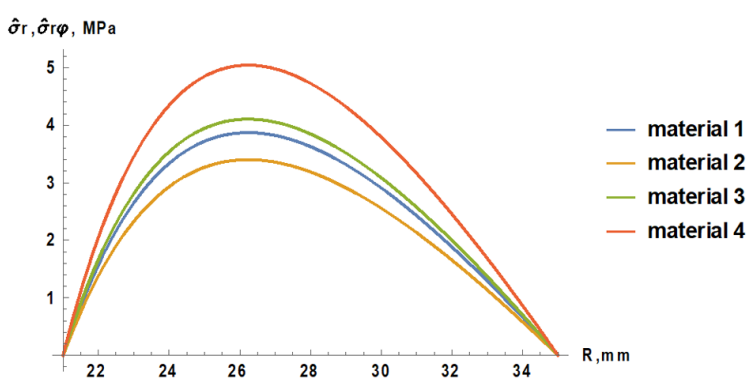

Fig. 4. Plots of the stresses $\hat{\sigma}_{r}=\hat{\sigma}_{r \varphi}$ as a function of the radius for the flat thermal problem

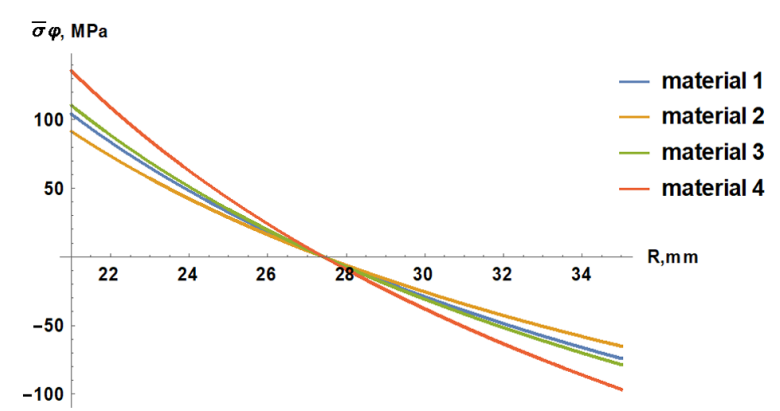

Fig. 3. Plots of the stresses $\bar{\sigma}_{\varphi}$ as a function of the radius for the axially symmetrical thermal problem

$\sigma_{B}=154 \mathrm{MPa}$ for the angle $\varphi=0$ and $r=a$, whereas for $\mathrm{r}=\mathrm{b}, 80 \mathrm{MPa}$ and $53 \mathrm{MPa}$, respectively.

The analysis conducted so far has been devoted to a variable temperature distribution on the outer plunger surface that corresponds better to an actual temperature distribution in the HPDC machine plunger. The assumed temperature distribution results in a complex stress state in the sleeve and the equivalent stresses defined according to the two hypotheses taken in the study. The maximal equivalent stresses $\sigma_{H M H}$ and $\sigma_{B}$ (according to the HMH and Burzynski hypotheses, correspondingly) for the inner $(r=a)$ and outer $(r=b)$ surface of the plunger, i.e., for the angle $\varphi=0$ according to (1), (18), (A1.5) and (A3.19) obtained in the study, are listed in Table 2.

The presented stresses have been compared to the equivalent stresses determined for the approximated axially symmetrical stress state, on the assumption of a constant temperature distribution on the inner and outer surface, as shown in [4]. According to relationships (1)-(3), it has been assumed that

$$
\begin{aligned}
& \widehat{T}(r=a, \varphi)=\bar{T}(r=a)=\bar{T}_{a}=150^{\circ} \mathrm{C} \\
& \widehat{T}(r=b, \varphi)=\bar{T}_{b}+\hat{T}_{b}=300^{\circ} \mathrm{C}
\end{aligned}
$$

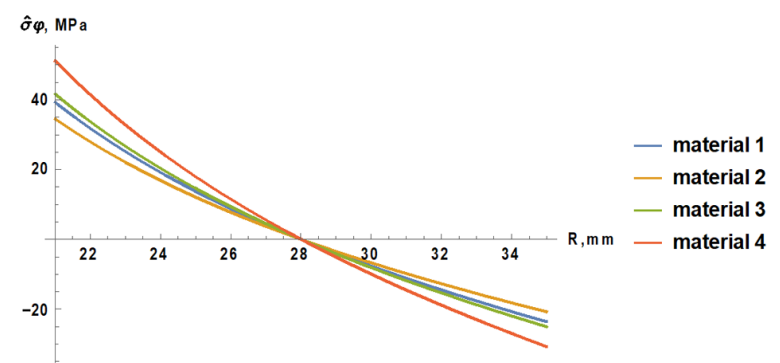

Fig. 5. Plots of the stresses $\hat{\sigma}_{\varphi}$ as a function of the radius for the flat thermal problem 


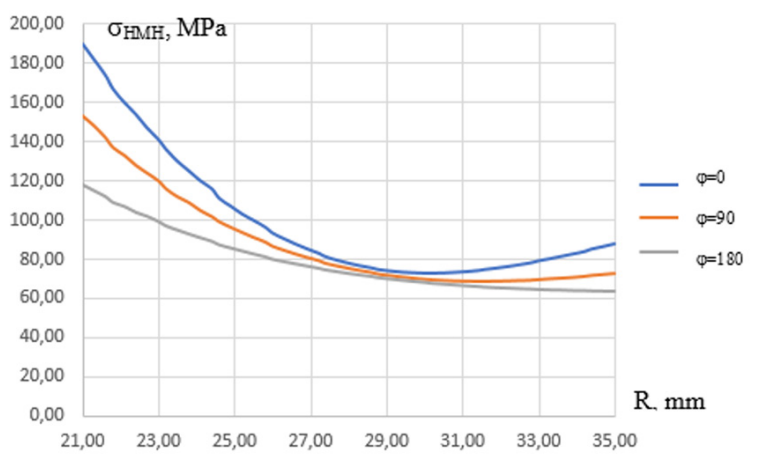

Fig. 6. Distribution of the equivalent stresses $\sigma_{H M H}$ for material 1

In Table 2, equivalent stresses according to the two hypotheses under analysis for the 3D stress state with the thermal axially symmetrical state and for the axial stresses in compression $\sigma_{z}$ (determined according to [4]), have been given as well. These stresses have been referred to as, $\widehat{\sigma}_{H M H}$ and $\widehat{\sigma}_{B}$, respectively.

As can be easily seen, the corresponding equivalent stresses for the simplified model [4] yield higher values than for the flat thermal state. The relevant ratios of the stresses $\hat{\sigma}_{H M H} / \sigma_{H M H}$, and $\hat{\sigma}_{B} / \sigma_{B}$ do not exceed 1.12. In the authors' opinion, it is a sufficient accuracy for estimation of a real stress state in comparison to the simplified model of the stress state assumed in [4].

\section{CONCLUSIONS}

An analysis of the stress state in HPDC machine plunger subjected to static mechanical loads and stationary thermal loads is presented. A variable temperature distribution on the outer surface of the plunger and a constant temperature inside the plunger due to forced cooling of its surface have been assumed. Four plunger material variants have been analysed. Components of the

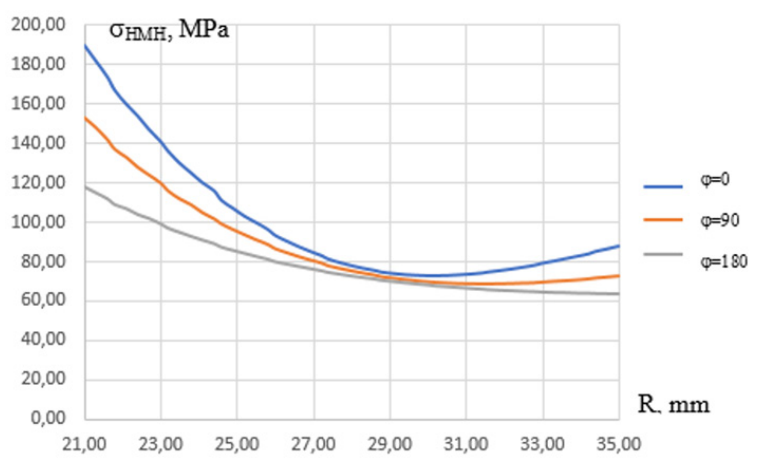

Fig. 8. Distribution of the equivalent stresses $\sigma_{H M H}$ for material 2

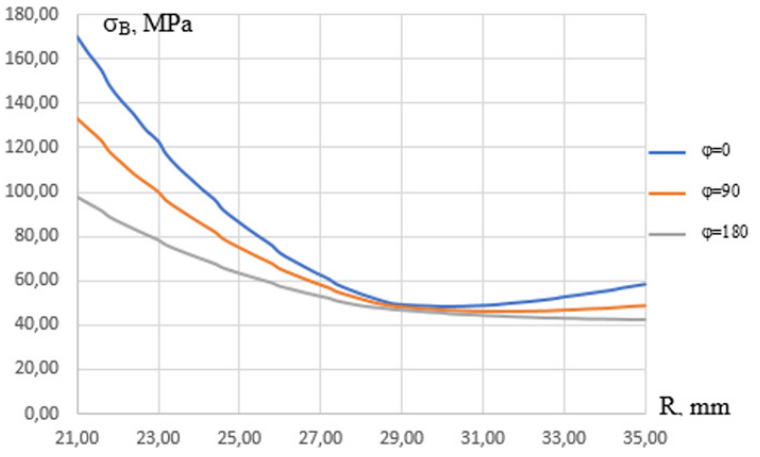

Fig. 7. Distribution of the equivalent stresses $\sigma_{B}$ for material 1

stress state, and then principal stresses in plungers have been determined, which has permitted equivalent stresses to be defined according to two strength hypotheses, including one accounting for various tensile and compression strengths of the plunger materials. It allows the equivalent stresses to be determined more adequately, which in turn can make a choice of the die casting plunger material more reasonable, by recognizing the operating conditions and providing thus higher wear and reliability of the plunger.

\section{Appendix I}

For the assumed axially symmetrical state of the thick-walled sleeve, the following formulas derived in [4] hold. For clarity, only four basic formulas are given in this Appendix, namely:

- equation of the heat conductivity in the stationary problem according to (1):

$$
\nabla^{2} T=\frac{1}{r} \frac{\partial}{\partial r}\left(r \frac{\partial T}{\partial r}\right)=0
$$

- equation of equilibrium according to (12):

$$
\frac{d \bar{\sigma}_{r}}{d r}+\frac{\bar{\sigma}_{r}-\bar{\sigma}_{\varphi}}{r}=0
$$

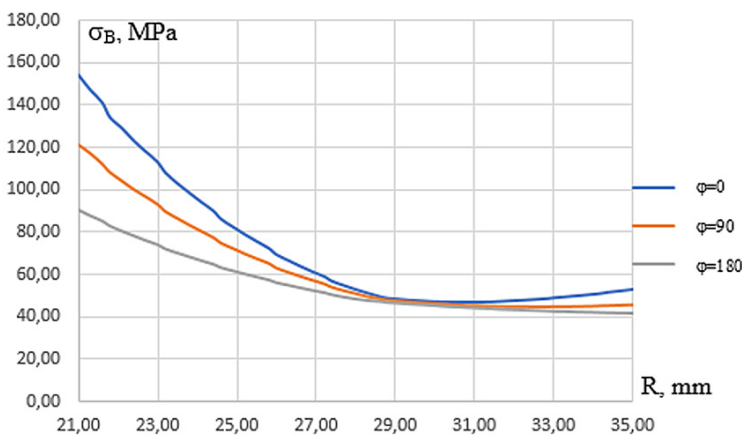

Fig. 9. Distribution of the equivalent stresses $\sigma_{B}$ for material 2 
Table 2. Equivalent stresses for the inner and outer side of the plunger

\begin{tabular}{|c|c|c|c|c|c|}
\hline \multirow{2}{*}{ Equivalent stresses } & \multirow{2}{*}{ Radius $r$} & Variant1 & Variant 2 & Variant 3 & Variant 4 \\
\hline & & \multicolumn{4}{|c|}{$\mathrm{MPa}$} \\
\hline$\sigma_{H M H}$ & $r=a$ & 190 & 174 & 198 & 231 \\
\hline$\sigma_{H M H}$ & $r=b$ & 87 & 80 & 92 & 111 \\
\hline$\sigma_{B}$ & $r=a$ & 170 & 154 & 178 & 212 \\
\hline$\sigma_{B}$ & $r=b$ & 58 & 53 & 61 & 74 \\
\hline$\hat{\sigma}_{H M H}$ & $r=a$ & 201 & 184 & 210 & 247 \\
\hline$\hat{\sigma}_{H M H}$ & $r=b$ & 97 & 87 & 103 & 125 \\
\hline$\hat{\sigma}_{B}$ & $r=a$ & 182 & 164 & 191 & 227 \\
\hline$\hat{\sigma}_{B}[4]$ & $r=b$ & 65 & 58 & 68 & 83 \\
\hline
\end{tabular}

- geometrical equations according to (13):

$$
\bar{\varepsilon}_{r}=\frac{d \bar{u}}{d r}, \quad \bar{\varepsilon}_{\varphi}=\frac{\bar{u}}{r}
$$

where: $\bar{u}$-radial displacement,

$\bar{\varepsilon}_{r}$ - radial strain,

$\bar{\varepsilon}_{\varphi}-$ circumferential strains,

$\bar{\sigma}_{r}-$ radial stresses

$\bar{\sigma}_{\varphi}-$ circumferential stresses,

$\bar{T}(r=a)=\bar{T}_{a}-$ temperature on the cylinder inner surface,

$\bar{T}(r=b)=\overline{T_{b}}-$ temperature on the cylinder outer surface, and, moreover,

$\bar{T}_{b}>\bar{T}_{a}$ and $\Delta \bar{T}=\bar{T}-T_{a}$.

A solution to (A1.1) for the sleeve is [4]:

$$
\bar{T}(r)=\bar{T}_{a}+\left(\bar{T}_{b}-\bar{T}_{a}\right) \frac{\ln r / a}{\ln b / a}
$$

Stress state components for the axially symmetrical state with boundary conditions (15) are expressed with the relationships [4]:

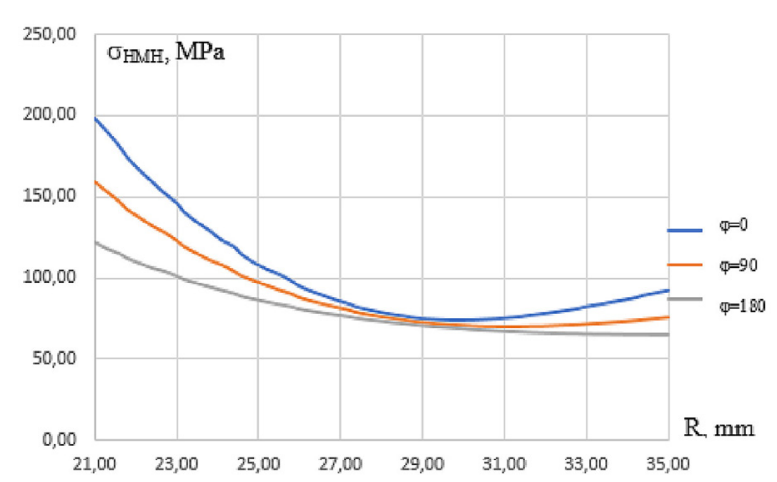

Fig. 10. Distribution of the equivalent stresses $\sigma_{H M H}$ for material 3

$$
\begin{aligned}
& \bar{\sigma}_{r}=-\frac{E \alpha\left(\bar{T}_{a}-\bar{T}_{b}\right)}{2}\left[\frac{\ln (b / r)}{\ln (b / a)}-\frac{\left(b^{2} / r^{2}-1\right)}{\left(b^{2} / a^{2}-1\right)}\right] \\
& \bar{\sigma}_{t}=-\frac{E \alpha\left(\bar{T}_{a}-\bar{T}_{b}\right)}{2}\left[\frac{\ln (b / r)-1}{\ln (b / a)}+\frac{\left(b^{2} / r^{2}+1\right)}{\left(b^{2} / a^{2}-1\right)}\right]
\end{aligned}
$$

\section{Appendix II}

The equation of heat conductivity in the flat stationary problem according to (1) is as follows:

$$
\frac{\partial^{2} \widetilde{T}}{\partial r^{2}}+\frac{1}{r} \frac{\partial \widetilde{T}}{\partial r}+\frac{1}{r^{2}} \frac{\partial^{2} \widetilde{T}}{\partial \varphi^{2}}=0
$$

with the boundary conditions

$$
\widetilde{T}(r=a, \varphi)=0 \text { and } \widetilde{T}(r=b, \varphi)=\hat{T}_{b}
$$

In the light of the above-mentioned, according to (2) and (8), a relationship

$$
\Delta \widetilde{T}=\widetilde{T}-\widetilde{T}_{a}=\widetilde{T}
$$

holds.

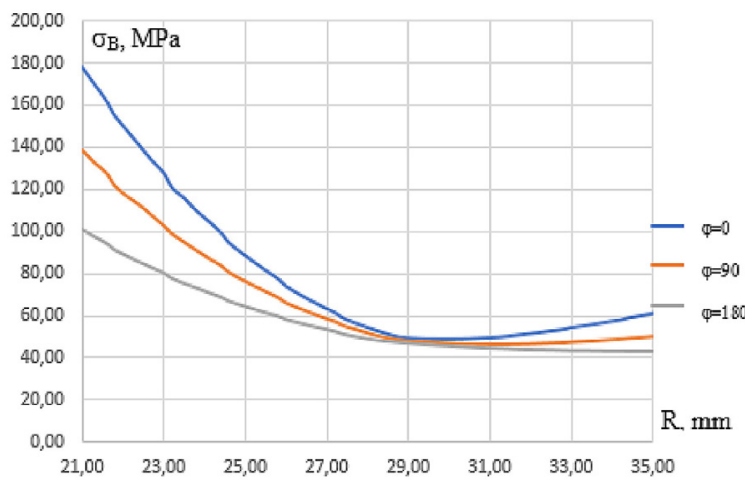

Fig. 11. Distribution of the equivalent stresses $\sigma_{B}$ for material 3 


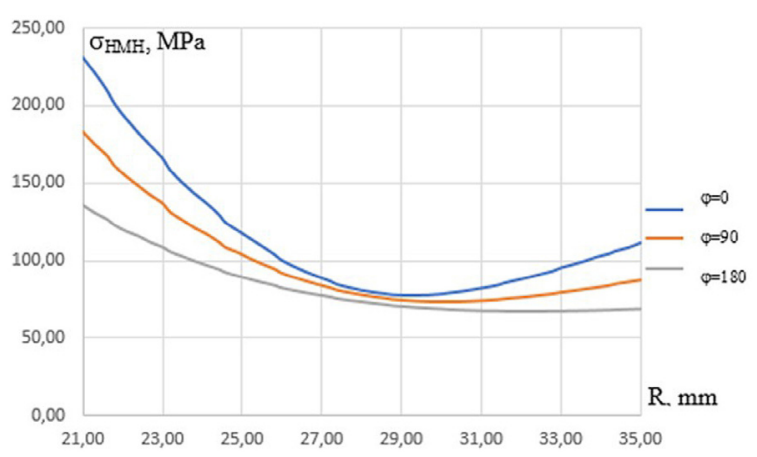

Fig. 12. Distribution of the equivalent stresses $\sigma_{H M H}$ for material 4

With respect to the temperature distribution assumed in (1):

$$
\widetilde{T}=\hat{T}(r) \cos \varphi
$$

a solution to (A2.1) is sought with the variable separation method in the form:

$$
\widetilde{T}=\hat{T}(r) \theta(\varphi)
$$

After differentiation and substitution of (A2.5) into (A2.1), we get:

$$
\frac{\frac{\partial^{2} \theta}{\partial r^{2}}}{\theta}=-\frac{\frac{\partial^{2} \hat{T}}{\partial r^{2}}+\frac{1}{r} \frac{\partial \hat{T}}{\partial r}}{\hat{T}} r^{2}=-k^{2}
$$

It follows from the first part of the equation that

$$
\frac{\partial^{2} \theta}{\partial r^{2}}+k^{2} \theta=0
$$

whose solution is a series of eigenvalues. However, in the light of (A2.4), i.e., for $\mathrm{k}=1$, we obtain:

$$
\theta=\cos \varphi
$$

After substitution of (A2.4) into (A2.1), a homogenous differential equation is attained:

$$
r^{2} \frac{\partial^{2} \hat{T}}{\partial r^{2}}+r \frac{\partial \hat{T}}{\partial r}-\hat{T}=0
$$

which is an Euler differential equation. For this type of equation, we seek a solution in the form $\hat{T}=r^{\beta}$. Taking into account the above solution, for the case when $\mathrm{k}=1$, it has the following form:

$$
\hat{T}=\frac{\hat{T}_{b} b}{b^{2}-a^{2}}\left(r-\frac{a^{2}}{r}\right)
$$

Taking account of (A2.5), (A2.8) and (A2.10) in (A2.4), the final solution is as follows:

$$
\widetilde{T}=\frac{\hat{T}_{b} b}{b^{2}-a^{2}}\left(r-\frac{a^{2}}{r}\right) \cos \varphi
$$

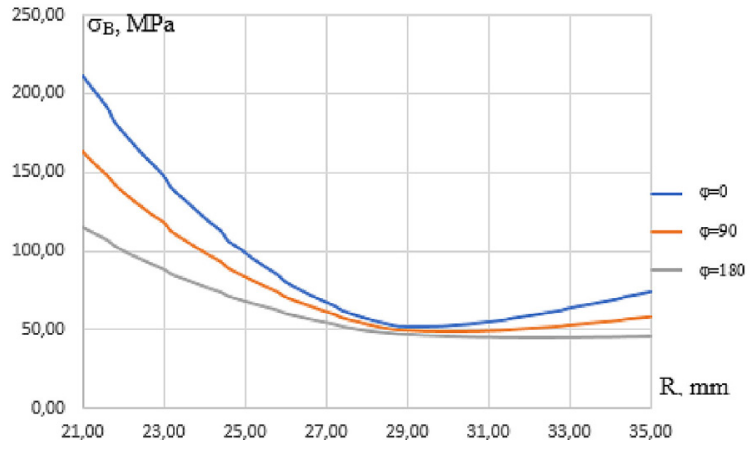

Fig. 13. Distribution of the equivalent stresses $\sigma_{B}$ for material 4

\section{Appendix III}

With respect to the assumed temperature distribution (A2.4), components of the stress state, according to (12), are as follows:

$$
\begin{aligned}
& \tilde{\sigma}_{r}(r, \varphi)=\hat{\sigma}_{r}(r) \cos \varphi \\
& \tilde{\sigma}_{r \varphi}(r, \varphi)=\hat{\sigma}_{r \varphi}(r) \sin \varphi \\
& \tilde{\sigma}_{\varphi}(r, \varphi)=\hat{\sigma}_{\varphi}(r) \cos \varphi
\end{aligned}
$$

The equations of equilibrium for the assumed flat stress state, corresponding to the stationary temperature distribution assumed in Appendix II (A2.11), according to (5) and (12) and boundary conditions (17), take the form:

$$
\begin{aligned}
& \hat{\sigma}_{r, r}+\frac{1}{r} \hat{\sigma}_{r \varphi, \varphi}+\frac{\hat{\sigma}_{r}-\hat{\sigma}_{\varphi}}{r}=0 \\
& \hat{\sigma}_{r \varphi, r}+\frac{1}{r} \hat{\sigma}_{\varphi, \varphi}+\frac{2}{r} \hat{\sigma}_{r \varphi}=0
\end{aligned}
$$

where the stress state components, according to (10), (12) and (13), are expressed with the relationships:

$$
\begin{aligned}
& \hat{\sigma}_{r}=\frac{E}{1-v^{2}}\left[\hat{u}_{, r}+\frac{v}{r} \hat{v}_{, \varphi}+\frac{v}{r} \hat{u}-(1+v) \alpha \hat{T}\right] \\
& \hat{\sigma}_{\varphi}=\frac{E}{1-v^{2}}\left[\frac{1}{r} \hat{v}_{, \varphi}+\frac{1}{r} \hat{u}+\hat{u}_{, r}-(1+v) \alpha \hat{T}\right] \\
& \hat{\sigma}_{r \varphi}=\frac{E}{2(1+v)}\left[\hat{v}_{, r}+\frac{1}{r} \hat{u}_{, \varphi}-\frac{\hat{v}}{r}\right]
\end{aligned}
$$

Substituting (A3.1) into (A3.2), we get:

$$
\begin{aligned}
& \hat{\sigma}_{r, r}+\frac{1}{r}\left(\hat{\sigma}_{r \varphi}+\hat{\sigma}_{r}\right)-\frac{1}{r} \hat{\sigma}_{\varphi}=0 \\
& \hat{\sigma}_{r \varphi, r}+\frac{2}{r} \hat{\sigma}_{r \varphi}-\frac{1}{r} \hat{\sigma}_{\varphi}=0
\end{aligned}
$$


Having summing up the sides, we have:

$$
\hat{\sigma}_{r, r}+\frac{1}{r} \hat{\sigma}_{r}=\hat{\sigma}_{r \varphi, r}+\frac{1}{r} \hat{\sigma}_{r \varphi}
$$

The above relation can be expressed also as:

$$
\frac{d}{d r}\left(r \hat{\sigma}_{r}\right)=\frac{d}{d r}\left(r \hat{\sigma}_{r \varphi}\right)
$$

Hence, it results that

$$
\hat{\sigma}_{r}=\hat{\sigma}_{r \varphi}
$$

The above reasoning leads to the statement that system of two equations (A3.1) is a linearly dependent system due to the fact that radial and tangential stresses are equal.

Returning to equations (A3.1), it can be said that the system of two equations has three unknown component functions of the stress state $\hat{\sigma}_{r}, \hat{\sigma}_{r \varphi}, \hat{\sigma}_{\varphi}$. With respect to (A3.3) and according to (13), the displacements have been assumed as:

$$
\begin{aligned}
\widetilde{u}(r, \varphi) & =\hat{u}(r) \cos \varphi \\
\widetilde{v}(r, \varphi) & =\hat{v}(r) \sin \varphi
\end{aligned}
$$

To solve explicitly these equations, the stresses have been expressed with the displacement components $\hat{u}, \hat{v}$ (13). After substitution of (A3.7) into (A3.2), we get:

$$
\begin{gathered}
\tilde{\sigma}_{r}=\hat{\sigma}_{r} \cos \varphi= \\
=\frac{E}{1-v^{2}}\left[\hat{u}+\frac{v}{r} \hat{v}+\frac{v}{r} \hat{u}-(1+v) \alpha \hat{T}\right] \cos \varphi \\
\tilde{\sigma}_{\varphi}=\hat{\sigma}_{\varphi} \cos \varphi= \\
=\frac{E}{1-v^{2}}\left[v \hat{u} \cdot \frac{1}{r} \hat{v}+\frac{1}{r} \hat{u}-(1+v) \alpha \hat{T}\right] \cos \varphi \\
\widetilde{\sigma}_{r \varphi}=\hat{\sigma}_{r \varphi} \sin \varphi=\frac{E(1-v)}{2\left(1-v^{2}\right)}\left[\hat{v}^{\bullet}-\frac{1}{r} \hat{v}-\frac{1}{r} \hat{u}\right] \sin \varphi
\end{gathered}
$$

where the following notations

$\hat{u}^{\bullet}=\hat{u}_{, r}, \hat{v}^{\bullet}=\hat{v}_{, r}$ are introduced.

Substituting (A3.8) into (A3.2), we obtain a system of equilibrium equations in displacements:

$$
\begin{gathered}
\hat{u^{\bullet \bullet}+}+\frac{\hat{u}^{\bullet}}{r}-\frac{3-v}{2 r^{2}} \hat{u}+\frac{1+v}{2 r} \hat{v}^{\bullet}-\frac{3-v}{2 r^{2}} \hat{v}= \\
=(1+v) \alpha \hat{T}_{b} \frac{b}{b^{2}-a^{2}}\left(1+\frac{a^{2}}{r^{2}}\right) \\
\frac{1-v}{2} \hat{v}^{\bullet}+\frac{1-v}{2 r} \hat{v}^{\bullet}-\frac{3-v}{2 r^{2}} \hat{v}-\frac{1+v}{2 r} \hat{u}-\frac{3-v}{2 r^{2}} \hat{u}= \\
=-(1+v) \alpha \hat{T}_{b} \frac{b}{b^{2}-a^{2}}\left(1-\frac{a^{2}}{r^{2}}\right)
\end{gathered}
$$

System of equations (A3.10) can be reduced to a system of first-order differential equations:

$$
\begin{gathered}
\hat{y}^{\bullet}+\frac{\hat{y}}{r}-\frac{3-v}{2 r^{2}} \hat{u}+\frac{1+v}{2 r} \hat{z}-\frac{3-v}{2 r^{2}} \hat{v}= \\
=(1+v) \alpha \hat{T}_{b} \frac{b}{b^{2}-a^{2}}\left(1+\frac{a^{2}}{r^{2}}\right) \\
\frac{1-v}{2} \hat{z}^{\bullet}+\frac{1-v}{2 r} \hat{z}-\frac{3-v}{2 r^{2}} \hat{v}-\frac{1+v}{2 r} \hat{y}-\frac{3-v}{2 r^{2}} \hat{u}= \\
=-(1+v) \alpha \hat{T}_{b} \frac{b}{b^{2}-a^{2}}\left(1-\frac{a^{2}}{r^{2}}\right) \\
\hat{u}^{\bullet}=\hat{y} \\
\hat{v}^{\bullet}=\hat{z}
\end{gathered}
$$

Next, a system of homogeneous equations (A3.11):

$$
\begin{aligned}
& \hat{y}^{\bullet}+\frac{\hat{y}}{r}-\frac{3-v}{2 r^{2}} \hat{u}+\frac{1+v}{2 r} \hat{z}-\frac{3-v}{2 r^{2}} \hat{v}=0 \\
& \frac{1-v}{2} \hat{z}^{\bullet}+\frac{1-v}{2 r} \hat{z}-\frac{3-v}{2 r^{2}} \hat{v}-\frac{1+v}{2 r} \hat{y}-\frac{3-v}{2 r^{2}} \hat{u} \\
& \hat{u}^{\bullet}=\hat{y} \\
& \hat{v}^{\bullet}=\hat{z}
\end{aligned}
$$

is to be solved.

We expect a solution to homogenous differential Euler equations (A3.12) as:

$$
\begin{aligned}
& \hat{u}(r)=k_{1} r^{\gamma} \\
& \hat{v}(r)=k_{2} r^{\gamma} \\
& \hat{y}(r)=k_{3} r^{\gamma} \\
& \hat{z}(r)=k_{4} r^{\gamma}
\end{aligned}
$$

After substitution of (A3.13) into (A3.12) and simplification of the equations with respect to $r^{\gamma}$ , the characteristic equation (zeroing condition of the determinant) has the following roots:

$$
\begin{array}{ll}
\gamma=0 & k_{2}=-k_{1} \quad \text { - double root } \\
\gamma=-2 & k_{2}=k_{1} \\
\gamma=2 & k_{2}=\frac{5+v}{1-3 v} k_{1}
\end{array}
$$

Taking the above-mentioned into consideration, a general solution to the system of equations (A3.12) has the form:

$$
\begin{aligned}
& \hat{u}(r)=A+B\left[\ln r-\frac{1+v}{2(3-v)}\right]+\frac{C}{r^{2}}+D r^{2} \\
& \hat{v}(r)=-A-B\left[\ln r+\frac{1+v}{2(3-v)}\right]+\frac{C}{r^{2}}+\frac{5+v}{1-3 v} D r^{2} \\
& \hat{y}(r)=\frac{B}{r}-\frac{2 C}{r^{3}}+2 D r \\
& \hat{z}(r)=-\frac{B}{r}-\frac{2 C}{r^{3}}+2 \frac{5+v}{1-3 v} D r
\end{aligned}
$$


Next, a particular solution to non-homogenous equation (A3.11) is sought with the method of variation of parameters:

$$
\begin{gathered}
\hat{u}(r)= \\
=A(r)+B(r)\left[\ln r-\frac{1+v}{2(3-v)}\right]+\frac{C(r)}{r^{2}}+D(r) r^{2} \\
\hat{v}(r)=. \\
=-A(r)-B(r)\left[\ln r+\frac{1+v}{2(3-v)}\right]+\frac{C(r)}{r^{2}}+\frac{5+v}{1-3 v} D(r) r^{2} \\
\hat{y}(r)=\frac{B(r)}{r}-\frac{2 C(r)}{r^{3}}+2 D(r) r \\
\hat{z}(r)=-\frac{B(r)}{r}-\frac{2 C(r)}{r^{3}}+2 \frac{5+v}{1-3 v} D(r) r
\end{gathered}
$$

The second term in the square bracket at $B(r)$ that occurs in the first two equations (A3.16) results from the singularity of the solution to the system of Euler equations (A3.11).

Substituting variated parameters (A3.16) into system (A3.11), we obtain a system of four equations with respect to the constants $\mathrm{A}(\mathrm{r}), \mathrm{B}(\mathrm{r}), \mathrm{C}(\mathrm{r})$ and $\mathrm{D}(\mathrm{r})$. After solving this system and integrating the expressions for derivatives of variated constants, the relationships for the constants A, $\mathrm{B}, \mathrm{C}$ and $\mathrm{D}$ are attained:

$$
\begin{aligned}
& A(r)=\frac{b \hat{T}_{b} \alpha(1+v)\left\{-\frac{r^{2}}{2}(-3+v)+\left[r^{2}(-3+v)+a^{2}(1+v)\right] \ln r\right\}}{4\left(a^{2}-b^{2}\right)(-1+v)}+A_{1} \\
& B(r)=\frac{b r^{2} \hat{T}_{b} \alpha(-3+v)(1+v)}{4\left(-a^{2}+b^{2}\right)(-1+v)}+B_{1} \\
& C(r)=\frac{b \hat{T}_{b} \alpha(1+v)\left[\frac{a^{2} r^{2}}{2}(-3+v)+\frac{r^{4}}{2}(1+v)\right]}{8\left(a^{2}-b^{2}\right)(-1+v)}+C_{1} \\
& D(r)=\frac{a^{2} b \hat{T}_{b} \alpha(-1+3 v)(1+v)}{16\left(a^{2}-b^{2}\right) r^{2}(-1+v)}+D_{1}
\end{aligned}
$$

where: $\mathrm{A}_{1}, \mathrm{~B}_{1}, \mathrm{C}_{1}$ and $\mathrm{D}_{1}$ - integration constants, which are to be determined from the boundary conditions. When the stress state components $\hat{\sigma}_{r}, \hat{\sigma}_{r \varphi}, \hat{\sigma}_{\varphi}$ are determined on the basis of displacement components (A3.15) and (A3.16), the integration constant $\mathrm{A}_{1}$ is reduced, i.e., it can be assumed that $A_{1}=0$. Thus, only three integration constants $\mathrm{B}_{1}, \mathrm{C}_{1}$ and $\mathrm{D}_{1}$ should be determined with respect to the four boundary conditions assumed (17). According to (A3.6), boundary conditions (17) have been modified:

$$
\begin{aligned}
& \hat{\sigma}_{r}(r=a)=\hat{\sigma}_{r}(r=b)=0 \\
& \int_{a}^{b} \hat{\sigma}_{r} d r=\int_{a}^{b} \hat{\sigma}_{r \varphi} d r
\end{aligned}
$$

which allows one to define explicitly the integration constants. When they are determined, the stress state components can be finally written as follows:

$$
\begin{aligned}
& \hat{\sigma}_{r}=\frac{a^{2} b E\left(a^{2}-r^{2}\right)\left(b^{2}-r^{2}\right) \hat{T}_{b} \alpha}{2\left(a^{4}-b^{4}\right) r^{3}} \\
& \hat{\sigma}_{\varphi}=-\frac{a^{2} b E\left[r^{2}\left(b^{2}-3 r^{2}\right)+a^{2}\left(b^{2}+r^{2}\right)\right] \hat{T}_{b} \alpha}{2\left(a^{4}-b^{4}\right) r^{3}} \\
& \hat{\sigma}_{r \varphi}=\frac{a^{2} b E\left(a^{2}-r^{2}\right)\left(b^{2}-r^{2}\right) \hat{T}_{b} \alpha}{2\left(a^{4}-b^{4}\right) r^{3}}
\end{aligned}
$$

\section{Appendix IV}

In [4], formulas for determination of equivalent stresses according to 4 strength hypotheses in a thick-walled sleeve were given for principal stresses. Below, following [4], the formulas in which notations used in this work occur are presented.

Having the principal stresses $\sigma_{1}^{*}, \sigma_{2}^{*}, \sigma_{3}^{*}$ determined, it is also possible to define equivalent stresses for two strength hypotheses, i.e., the Huber-Mises-Hencky hypothesis (HMH) $[1,3,9]$ and the Burzynski theories [9], because the plunger material can have various maximal allowable stresses in tension $k_{r}$ and maximal allowable stresses in compression $k_{c}$ (or the tensile strength $R_{m}$ and the compression strength $R_{c}$, correspondingly).

For isotropic materials, equivalent stresses are determined according to:

- Huber-Mises-Hencky hypothesis (denoted as $\mathrm{HMH})[1,3,9]$

$$
\begin{gathered}
\sigma_{\text {HMH }}=\left(\sigma_{\text {eqv }}\right)_{H M H}= \\
=\sqrt{\frac{1}{2}\left[\left(\sigma_{1}^{*}-\sigma_{2}^{*}\right)^{2}+\left(\sigma_{2}^{*}-\sigma_{3}^{*}\right)^{2}+\left(\sigma_{3}^{*}-\sigma_{1}^{*}\right)^{2}\right]} \leq k_{r}
\end{gathered}
$$

- Burzynski hypothesis (denoted as B) [9]

First, two auxiliary quantities should be found:

$$
\omega_{1}=\left(\sigma_{1}^{*}+\sigma_{2}^{*}+\sigma_{3}^{*}\right) / 3
$$

$$
\omega_{2}=\sqrt{\left(\sigma_{1}^{*}-\sigma_{2}^{*}\right)^{2}+\left(\sigma_{2}^{*}-\sigma_{3}^{*}\right)^{2}+\left(\sigma_{3}^{*}-\sigma_{1}^{*}\right)^{2}} / 3
$$

They allow for determination of equivalent stresses according to the presented hypothesis for three ranges, namely:

o when $\quad-\sqrt{2} \leq \omega_{2} / \omega_{1} \leq 0, \quad$ then
$\sigma_{B}=\left(\sigma_{\text {eqv }}\right)_{B}=3 \omega_{2} /(\sqrt{2} z)<k_{r}$ 
o when $\omega_{2} / \omega_{1}<-\sqrt{2}$ or $\sqrt{2<} \omega_{2} / \omega_{1}$, then

$$
\sigma_{B}=\left(\sigma_{e q v}\right)_{B}=
$$

$=3(z+1) \omega_{2} /(2 \sqrt{2} z)+3(z-1) \omega_{1} /(2 z)<k_{r}$

o when $0 \leq \omega_{2} / \omega_{1} \leq \sqrt{2}$, then $\sigma_{B}=\left(\sigma_{e q v}\right)_{B}=3 \omega_{2} /(\sqrt{2} z)+3(z-1) \omega_{1} / z<k_{r}$

where: $z=R_{c} / R_{m}=\left(k_{c} / k_{r}\right)$

In the case when $z=1$, the Burzynski hypothesis is reduced to the HMH hypothesis.

The determination of mechanical and thermal stress components and equivalent stresses enables a parametric analysis and facilitates a possible evaluation of tribological properties of the HPDC machine sleeve.

\section{REFERENCES}

1. Brzoska Z. Wytrzymałość materiałów. PWN, Warszawa 1983.

2. Derski W. Podstawy teorii sprężystości. PWN, Warszawa 1965.
3. Dyląg Z., Jakubowicz A., Orłoś Z. Wytrzymałość materiałów. WNT, Warszawa 2013.

4. Jankowski J., Kołakowski D., Pisarek B.P.. Analysis of mechanical and thermal stresses in a pressure casting machine plunger. Advances Science and Technology Research Journal, 2020 (In print).

5. Kanlıkama B., Abuşoğlu A., Güzelbey I.H. Coupled thermoelastic analysis of thick-walled pressurized cylinders. International Journal of Energy and Power Engineering, 2(2), 2013, 60-68.

6. Qi Shi M.S. Prediction of Thermal Distortion and Thermal Fatigue in Shot Sleeves. Dissertation, Advisor: Miller A. Presented in Partial Fulfillment of the Requirements for the Degree Doctor of Philosophy in the Graduate School of The Ohio State University, USA, 2002.

7. https://etd.ohiolink.edu/!etd.send_file?accession= osu1034967167\&disposition=inline Silva E., Úbeda V. \& Fischer W. Shot Sleeves for Die Casting (Thermal Variations and its Consequences) Proceedings of the Foundry Congress: CONAF 2009, 22-25 September 2009 in Sao Paulo, Brazil 2009. http://wfischer.com.br/en/files/CONAF-paper.pdf

8. Timoshenko S., Goodier J.N. Teoria sprężystości. Wydawnictwo Arkady, Warszawa 1962.

9. Walczak J. Wytrzymałość materiałów oraz podstawy teorii sprężystości i plastyczności. PWN, Warszawa-Kraków 1978. 